Background and Aims PICU provides high level of care to critically ill or injured children. Our aim was to analyse the socioeconomic characteristics of their families.

Methods 127 children, hospitalized from 10/05/2008 to 10/5/2009, were analyzed retrospectively: age, gender, parents' age, nationality, residence, marital status, number of childrens, parents' education, employment, insurance, and way of transportation, and were correlated with the days of hospitalization, severity scores and outcome. Data analysis with the SPSS 17

Results $73(57.5 \%)$ were males and 54 (42.5\%) girls, mean age 4.5 and 6.7 years respectively. $78.2 \%$ were from this country, and $4.1 \%$ tourists, $17.5 \%$ immigrants and $2.3 \%$ gypsy. No significant difference in the distribution for gender, but a statistically significant difference in educational level of both parents, in relation to nationality $(p<0,011) .75 \%$ foreign and $10 \%$ Greeks parents were primary schools, $15 \%$ and $70 \%$ high school and only $7 \%$ and $20 \%$ university graduates, respectively. Higher TISS 28 and TISS 76 (worst condition) in foreigners. Higher PRISM and PRISM Predicted Mortality (\%) severity scores (more serious condition), higher TISS 28 and TISS 76 and more days of hospitalization in children transported by ambulance/airplane. Foreigner children, transferred by ambulance or airplane, had the higher death rate.

Conclusions The severity of illness, duration of hospitalization and outcome, appear to be related to the socioeconomic characteristics of families and the way of transportation.

\section{EPIDEMIOLOGY OF SEVERE TRAUMATIC BRAIN INJURY (TBI) IN PEDIATRIC POPULATION OF THE WEST OF ALGERIA}

doi:10.1136/archdischild-2012-302724.1740

H Bouguetof, MA Negadi, K El Halimi, D Boumendil, ZC Mentouri. Pediatric Intensive Care Unit, Faculty of Medicine - Oran University, Oran, Algeria

Background and Aims Algeria is in the fourth rank of road accidents in the world.

The Aim of the study was to provide recommendations to government and health authorities, based on hard evidence, for improving health care delivery to children with severe TBI in the referral region of the university hospital center in Oran.

Method In this cohort, all children with severe TBI was admitted to the Paediatric Intensive Care Unit (PICU) of the University Hospital Centre of Oran, between the January 1, 1995 and December 31, 2007

Results The average age is 7 years $1 / 2$. Road accidents are in the origin of more than $70 \%$ of the cases in which $80 \%$ are pedestrians. $65 \%$ of pedestrians are injured on roads in rural environment (countryside). The pre hospital management is unfortunately almost absent. $1 / 3$ of children had GCS score $<5$. Median Injury Severity Scale (ISS) score was 26 (IOR 21-33; Range 9-75). The polytraumatism represents $1 / 5$ of the cases. The cerebral œedema was found in $3 / 4$ of the cases. On the 573 children, the rate of survival is $61 \%$. In this study, the road accidents represent the main cause of severe TBI in children and are responsible of a more high mortality essentially due to the absence of prehospital management.

Conclusion The improvement of prehospital management must be reduce the mortality and improve the prognosis of severe TBI and the information of the population should be promoted to reduce the frequency of severe TBI.

\section{PULSE OXIMETRY IN HEALTHY NEWBORNS AFTER MIDWIFERY SUPERVISED UNCOMPLICATED HOME BIRTHS; USE OF INTERNATIONAL ACCEPTED PERCENTILES}

doi:10.1136/archdischild-2012-302724.1741
${ }^{1} \mathrm{M}$ Smit, ${ }^{2} \mathrm{~A}$ Ganzeboom, ${ }^{3} \mathrm{~J}$ Dawson, ${ }^{2} \mathrm{AT}$ Pas. ${ }^{1}$ Obstetrics and Gynaecology: ${ }^{2}$ Neonatology, Leiden University Medical Center, Leiden, The Netherlands; ${ }^{3}$ Neonatology Research, The Royal Women's Hospital, Melbourne, VIC, Australia

Background Percentiles of oxygen saturation as a function of time from birth in uncompromised infants born at term are now defined. However, in these percentiles infants born after assisted deliveries (ventouse, forceps), by cesarean, after augmentation of labour, or epidural analgesia were also included.

Aim To evaluate if international accepted percentiles of neonatal oxygen saturation and heart rate values are applicable in infants born after non-intervention vaginal deliveries.

Methods During ten consecutive months, 27 midwives in the Leiden region used a Masimo pulse oximeter and perform measurements directly after birth infants born after non-intervention vaginal deliveries. Data was stored and analyzed using the skewness-median-coefficient of variation (LMS) method.

Results During the study period oximetry was recorded in 101 births. Percentiles of oxygen saturation and heart rate are shown in figure 1 and 2 . The percentiles are comparable to the international accepted values, except for the first 3 minutes for oxygen saturation and the first minute for heart rate.

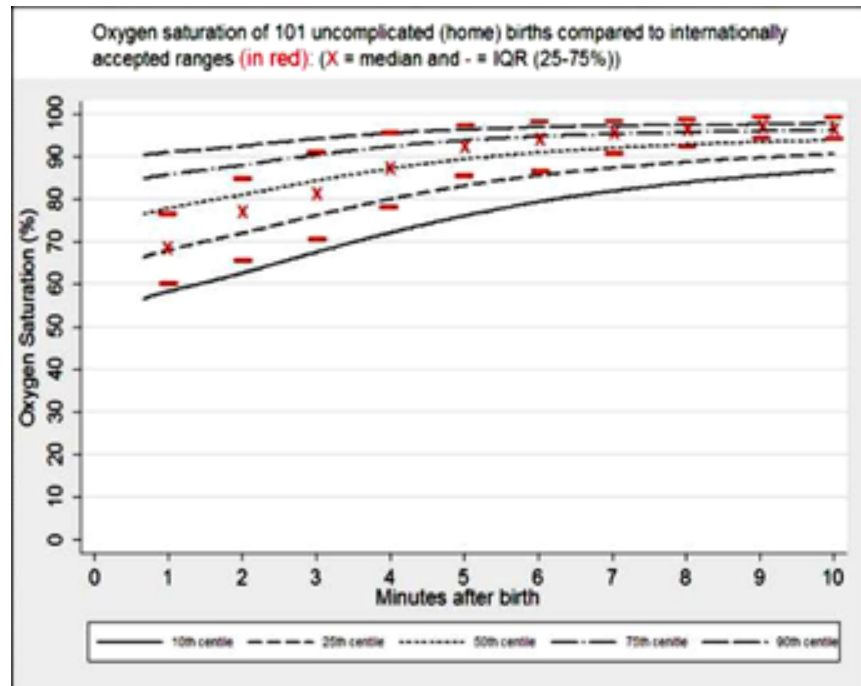

Abstract 1741 Figure 1

Neonatal heart rates of 101 uncomplicated (home) births compared to internationaly accepted ranges (in red): $(X=$ median and $-=\operatorname{IQR}(25-75 \%))$

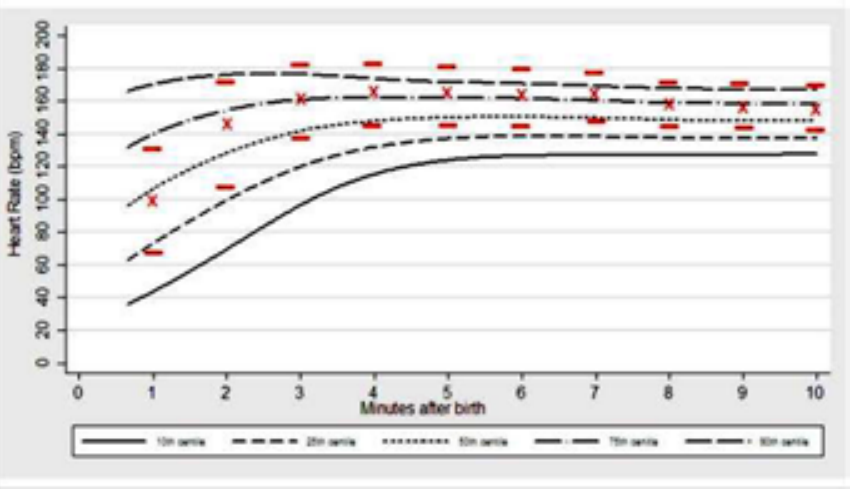

Abstract 1741 Figure 2

Conclusions The accepted percentiles for heart rate and oxygen saturation are applicable to infants born after a non-intervention 